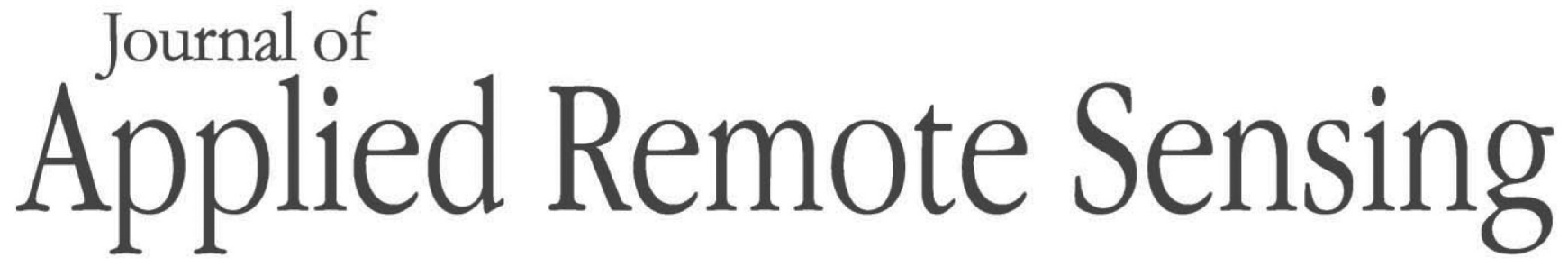

\title{
Sensorized robotic sphere for large exterior critical infrastructures supervision
}

Juan David Hernández

David Sanz

Gonzalo R. Rodríguez-Canosa

Jorge Barrientos

Jaime del Cerro

Antonio Barrientos 


\title{
Sensorized robotic sphere for large exterior critical infrastructures supervision
}

\author{
Juan David Hernández, David Sanz, Gonzalo R. Rodríguez-Canosa, \\ Jorge Barrientos, Jaime del Cerro, and Antonio Barrientos \\ Centre for Automation and Robotics, Universidad Politécnica de Madrid-Consejo Superior de \\ Investigaciones Científicas, Madrid, Spain \\ antonio.barrientos@upm.es
}

\begin{abstract}
The surveillance and inspection tasks in large exterior critical infrastructures have arisen as critical processes. More complex challenges are now present, and the traditional approaches are sometimes obsolete for facing these new menaces. An alternative system-a mobile sensor, spherical shaped - that provides a flexible, versatile, and reliable way to perform measurements is proposed. Even more, thanks to its original traction method (based on center of gravity destabilization), the system has a result as an all-terrain vehicle that guarantees a safe and friendly interaction with the environment. It has been widely tested, verifying as well the accurate acquisition performance, resulting in this system as a suitable sensing and monitoring alternative. (C) 2013 Society of Photo-Optical Instrumentation Engineers (SPIE) [DOI: 10.1117/1.JRS.7 $.073522]$
\end{abstract}

Keywords: critical infrastructures; robotic sphere; sensors; supervision; security; surveillance.

Paper 12422 received Nov. 26, 2012; revised manuscript received Jun. 14, 2013; accepted for publication Jul. 9, 2013; published online Aug. 19, 2013.

\section{Introduction}

Critical infrastructures (CIs) are those physical and information technology facilities, networks, services, and assets which, if disrupted or destroyed, would have a serious impact on the health, safety, security, or economic well-being of citizens or on the effective management, and governance of a country. ${ }^{1}$ Their security and effective surveillance have become challenging requirements that must be taken into account when designing the operation and integrated functioning of the essential elements of the installation.

Exterior critical infrastructures (ECIs) present common characteristics, mainly their size (i.e., usually quite large) and location (i.e., commonly far away from highly populated areas), that permit to group and study their security and surveillance under a common scheme. Power plants, communication centers, energy production plants, dangerous material storage facilities, and dams are examples of ECIs.

In most ECIs, the security and surveillance tasks have been usually undertaken by a combination of static sensors (cameras, movement detectors, etc.) and human guards. In this context, the use of robotic solutions is becoming quite popular due to their inherent advantages in terms of (1) intensification, (2) larger perception range, (3) greater mobility and adaptability, and (4) risk reduction for human guards. From these factors, the reduction of risk to human guards has become the main thrust for the implementation of these type of surveillance solutions.

Different types of robots and robotic solutions have been designed and used according to specific scenarios. From the late 1980s, when the IRIS (industrial remote inspection system) robot performed inspection tasks in nuclear plants, ${ }^{2,3}$ to current commercial robotic surveillance solutions (e.g., the patrolling of South Korean's Pohang prison by Roboguard ${ }^{4}$ ), security robots have been successfully deployed. In this context, in the most advanced sensing systems, not only ground robots ${ }^{4-6}$ but unmanned aerial vehicles (UAVs), have been incorporated as part of security and surveillance multirobot systems (MRSs). ${ }^{7,8}$ Examples of these platforms are the

0091-3286/2013/\$25.00 ㄷ 2013 SPIE 
AirRobot's AR100-B (http://www.airrobot.com/index.php/products-28.html) and Astec's Pelican (http://www.asctec.de/asctec-pelican-3/) UAVs.

Nevertheless, the particular ECI conditions make difficult to provide a generic solution: uncertain terrain conditions, presence of humans, different significant magnitudes, or great variability in the area-to-cover supposes a real challenge. Even more considering that some facilities could suppose a risk for the robot's own safety (e.g., radiation in nuclear plants). Therefore, a mobile monitoring solution for general ECIs would require the incorporation of robotic vehicles able to displace themselves on rough terrains over long distances, carry different payloads, and avoid collisions and dangers. In this scenario, spherical robots arise as a perfect compromise capable of addressing most possible contingencies. Although rarely used for this type of applications, ${ }^{10}$ it is our belief that their use may be an effective alternative to more classic robots.

The main objective of this work is to propose a generic surveillance system-based on spherical robots-able to measure the required magnitude in any kind of ECI scenario. In this sense, the present article exposes this work. It is organized as follows. Section 2 outlines the specific requirements of a surveillance robot for this infrastructure and evaluates the possible solutions to the problem. Section 3 presents and analyzes the available spherical robot solutions. In Sec. 4, four experiments are presented and discussed. Finally, Sec. 5 summarizes the conclusions and future work in this matter.

\section{Problem Outline and Solution Assessment}

This section presents a study of the characteristics of an ECI, as well as a summary of the requirements of a surveillance robotics sensing platforms working in this environment. Finally, different robotic platforms for these applications are critically studied and compared.

\subsection{General Characteristics of an ECl}

ECIs present a set of characteristics that justify the incorporation of a specifically designed MRS for security and surveillance. Safety and robustness are the main requirements for the use of robots in these sensitive facilities, where the failure of any subsystem might cause serious damages to the facility and its surroundings. Thus, reliability of the robotic solution must be warranted by all means.

The main ECI features to be taken into account to design a surveillance MRS solution are the following:

- Location: The CI locations can be quite different, but in general they are placed away from population centers. Very often, these facilities will be found in industrial areas in the suburbs of the cities, as well as in zones far away from the urban nuclei.

- Size: They are usually large facilities with varying sizes from 60 to $3000 \mathrm{Ha}$ depending on the type of ECI.

- Surface: In general, part of the whole installation grounds will be asphalted with tracks, passages, or streets to move through its interior. Depending on the size of the facility, these ways or roads will be of a greater or smaller size and will be paved in a better or worse way. In installations integrated in rural terrains placed far away from urban areas, the ways inside the installation will usually be made of compacted soil.

- Inclination: Generally, there will not be pronounced slopes within the facility, although there can be several height levels. Most often in the installation there will be two or more parts placed at quite different levels. In this case, the parameter "slope" must be taken into account to define the traction system of the robots.

- Internal Elements: Main internal elements in this type of infrastructure are large storage buildings and loading docks. The presence of human operators and other machines and vehicles should also be expected.

The previously mentioned characteristics describe general characteristics of an ECI. Focusing in different types of infrastructures some differences can be noticed. A summary of the characteristics for different types of ECI is shown in Table. 1. 


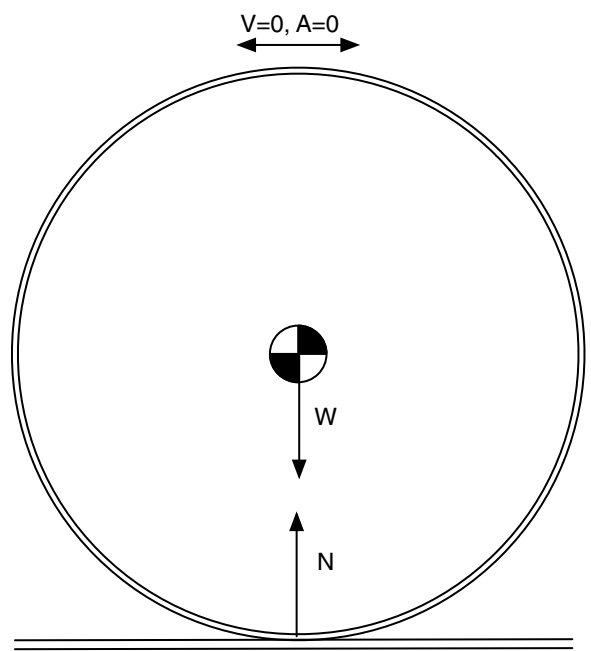

(a)

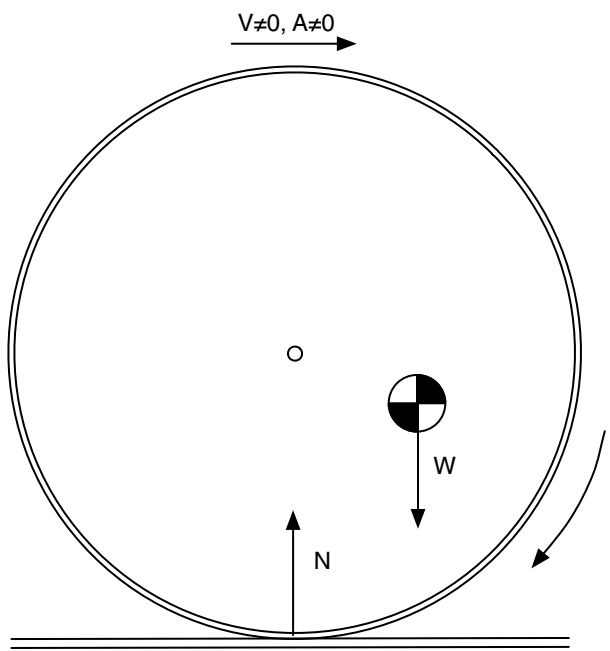

(b)

Fig. 1 Basic principle of motion. The projection of the center of mass over the surface may define whether the sphere has nonzero acceleration and velocity. (a) Balanced configuration. (b) Unbalanced configuration.

The surveillance of any ECI concerns two main aspects: the security against external threats and the security against internal malfunctions. For both of these tasks, it is important to have agile sensing platforms capable of moving through the whole facility without disturbing its normal operation. In addition, other important inspection tasks of the robots refer to the monitoring of the damage of internal elements (lamps, fences, etc.) or vegetation growth monitoring.

This article is focused on the requirements of a robotic sensing platform to monitor different parameters along with the infrastructure. These requirements are presented in the next section.

\subsection{Requirements of Robotic Sensing Platform for ECI Surveillance}

The most important characteristic to design a robot intended for an ECI monitoring is its reliability and robustness. They imply that the robot must remain stable and provide the same performance regardless of the external conditions, at both the hardware and software levels.

The ECI location, typically in remote scenarios, may result in exposing the robots to hard conditions such as heavy rains, extreme temperatures, or dusty winds. It can also be expected that a part of the installation is made of rough terrain and that the different weather conditions along with the different seasons of the year alter significantly the conditions of the environment. For example, there can be snow or ice sheets in winter, puddles or muddy areas in autumn, or sandy and crumbly grounds during summer.

All these factors affect directly the design of the movement mechanism, probably the most important element of the robot. Moreover, the traction system needs to be versatile and able to displace the robot along with the different types of surfaces, from urbanized even terrains where the robot might slide to rough nonstructured surfaces. The traction system should allow the robot to follow trajectories with sharp angles and narrow passages.

Due to the size of the infrastructure, another important factor is the autonomy of the sensing platform. These robotic platforms should be able to perform continuous monitoring for several hours without recharging.

The internal elements of the infrastructure also affect the choice of the traction system and the general design of the robot. The chosen vehicle should be able to perform its inspection operation ensuring both its own safety and the safety of the rest of the elements. Since human workers are to be expected in the facility, a friendly interaction system is necessary. Thus, the robot should be able to detect and avoid static and mobile objects or, in case of collision, not injure the human operators or damage any part of the infrastructure.

Additional minor considerations such as the ability of the cameras of not being affected by dust when taking images should also be considered in the design of a robotic solution. 
Table 1 Summary of characteristics of different types of $\mathrm{Cl}$.

\begin{tabular}{lccccc}
\hline \hline ECl type & Location & Size & Surface & Inclination & Internal elements \\
\hline Basic & Nonurban & 100 to $3000 \mathrm{Ha}$ & Asphalt & Small & Multiple \\
Solar & Nonurban & 60 to $120 \mathrm{Ha}$ & Sand and soil & Moderate & Heliostats \\
Nuclear & Nonurban & 60 to $100 \mathrm{Ha}$ & Irregular soil & Flat & Towers \\
Airport & Semi-urban & $3000 \mathrm{Ha}$ & Compacted soil & Flat & Control tower \\
Harbor & Urban & $600 \mathrm{Ha}$ & Asphalt & Flat & Containers \\
Dam & Nonurban & 500 to $2000 \mathrm{~m}$ & Asphalt & Great slopes & - \\
\hline \hline
\end{tabular}

In summary, the most important characteristics of robotics sensing platform are: robustness, ability to move in different terrains, autonomy, and safe interaction with the environment.

\subsection{Analysis of Present Solutions}

The classical solution to detect radioactive leakages, present in all nuclear facilities, is a network of sensors distributed along with the whole installation. The main disadvantage of this method is that, to detect a radiation leakage, it must be significant enough to be detected by the nearest sensor. Small or localized leakages would remain undetected for a long period of time if the leakage is located far from a sensor. Increasing the number of sensors to a point where this factor is no longer significant would be extremely expensive and inefficient.

A more efficient approach would be to use a robotic solution. Different types of solutions with different configurations and characteristics have been studied and evaluated with regard to their use for localized radioactive leakage detection in an nuclear waste residues (NWR). To systematically perform this analysis different parameters have been defined according to the requirements specified in Sec. 2.2. These characteristics are: mobility, maneuverability, robustness, autonomy, payload capacity, action range, measurement accuracy, and interaction with the other elements of the installation.

- UAV: These vehicles present a high level of mobility, but their low autonomy, small payload capability, and poor maneuverability makes them unsuitable for the selected application. Furthermore, since the leakages mostly occur at the ground level, the advantages of having an aerial perspective are lost as the UAVs would have to fly close to the ground for a reliable detection. Other problems with this type of vehicles are their inability to operate in extreme meteorological conditions (i.e., heavy rain or gusty winds), special requirements for take-off and landing, and security concerns in their interaction with the other elements (i.e., dangerous situations for the human operators in case of robot malfunctions). Their expected high initial and current maintenance costs are additional factors that preclude the use of these vehicles for this application.The UAVs can be classified into two different types with specific characteristics:

- Rotational-wing UAV: This type of UAV has usually a slightly smaller payload capacity and robustness but a higher maneuverability.

- Fixed-wing UAV: The main advantage of this type of UAV is its higher action range and autonomy.

- Large UGV: The main problem of this type of unit is their size, as large UGVs have low maneuverability and mobility and cannot fit through narrow passages. Although other characteristics of these vehicles, such as a large autonomy, big payload capacity, high robustness, and high measurement accuracy, are very desirable for the intended application, the size limitation usually discards this type of robot for surveillance applications in NWR facilities.

- Small UGVs team: Using a robotic swarm of small simple robots is actually very suitable for this application. Its main advantages are the ability to perform simultaneous measurements in different parts of the installation and the great maneuverability and mobility that 
present this type of robots. Their small size fulfills a critical requirement since it allows them to fit through narrow spaces and to follow sharp angled trajectories. Also, in case of collision, they would cause minimum impact to the other elements of the environment. Their main drawback refers to their limited payload capacity that imposes the use of small and light batteries, thus reducing their autonomy and their autonomous field of work.

- Bio-inspired systems: The main limitations of bio-inspired locomotion systems reside in the characteristics of their movement systems (e.g., leg based or crawling methods) that, in comparison with other more conventional methods (e.g., wheeled propulsion), are usually highly power consuming and provide low velocities. The available commercial and laboratory-based bio-inspired platforms are still highly unstable and are unable to cover a large area in a reasonable period of time.

A suitable solution that combines great mobility and maneuverability together with an acceptable autonomy, high payload capacity, and robustness is a robotic sphere similar to that presented by Seeman et al. ${ }^{10}$ or Zhan et al. ${ }^{11}$ The main benefits of this type of robots come from their shape and motion structure that minimize energy losses due to friction. Their spherical shape allows the robot to displace themselves along different terrains and surfaces (even water) with a minimum consumption of energy. The motion principle consists of a pendulum-based device that induces movement destabilizing the sphere. This principle is different than that of more traditional movement methods where a torque momentum is responsible to generate displacement in wheels.

This type of movement generation, implying a minimum lift over the background surface, allows the robot to move without producing dust. This beneficial aspect of its operation is not significantly affected by the payload weight as long as it is balanced within the sphere. The shape of the robot and its relatively small size and low weight imply a friendly interaction with the environment since any collision would have a minimum effect on the other elements of the installation. Another advantage is the rapid recuperation of its attitude in case of destabilization, i.e., a recovery effect similar to that of tilting toys.

The same characteristics (i.e., shape, size, and movement control), which are so advantageous with regard to the implementation of these robots in an NWR, have a negative impact in the robot control and navigational systems. Precise trajectories, locations, or attitudes are difficult to define and follow, resulting sometimes in curvilinear uncontrolled trajectories. When static, the robot contacts the surface in a single point, generally resulting in an unstable situation. Big obstacles would also be a problem. Nevertheless, these two limitations are not critical in the present scenario where huge obstacles are not expected and high accuracy in the attitude is not needed.

A critical comparison of the aforementioned vehicle solutions with respect to the use of the robotic sphere is summarized in Table 2 .

In the next section, the internal mechanism of the proposed robotic sphere is explained in more detail.

Table 2 Comparison of available solutions for radioactive leakage detection according to the following parameters: $C_{1}=$ mobility, $C_{2}=$ maneuverability, $C_{3}=$ robustness, $C_{4}=$ autonomy, $C_{5}=$ payloadcapacity, $C_{6}=$ actionrange, $C_{7}=$ safe interaction with other elements.

\begin{tabular}{lccccccc}
\hline \hline & $C_{1}$ & $C_{2}$ & $C_{3}$ & $C_{4}$ & $C_{5}$ & $C_{6}$ & $C_{7}$ \\
\cline { 2 - 8 } Sensor networks & $\mathrm{x}$ & $\mathrm{x}$ & ++ & +++ & $\mathrm{x}$ & ++ & +++ \\
\hline RW UAV & +++ & +++ & + & + & ++ & ++ & + \\
FW UAV & +++ & + & ++ & ++ & +++ & +++ & + \\
Large UGV & + & + & +++ & +++ & +++ & ++ & + \\
Small UGVs team & ++ & ++ & ++ & ++ & + & + & ++ \\
Bio-inspired & + & ++ & + & + & + & + & ++ \\
Robotic sphere & ++ & +++ & +++ & +++ & + & ++ & +++ \\
\hline \hline
\end{tabular}




\section{System Outline}

Considering the aspects mentioned above, a rolling robot has been proposed as a solution to fulfill successfully all the operational requirements. Its maneuverability, versatility, and capacity to recover from collisions are characteristics that make this type of robot suitable for the particular application studied in this article. Throughout this section, the main features of the system are explained, including the mathematical concepts involved in its operation, as well as the main mechanical and electronic design aspects of current prototypes.

\subsection{Basic Principle of Locomotion}

The objective of this work is to present a rolling robot with a spherical shape, called robotic sphere (ROSPHERE), as an alternative mobile platform to perform monitoring and inspection tasks. In contrast to other mobile robots (e.g., walking systems) whose basic locomotion principle is the system stability, movements in robotic spheres are induced by instability. Another consequence is that, due to its regular shape, the robot recovers easily from collisions so that, regardless the direction of the impact, the robot always tends to fall into a recoverable configuration. Herein, in order to have a global view of the robot capabilities, we will analyze the internal mechanism which endows the system with these characteristics.

Let us consider first a sphere where mass is uniformly distributed. In this case, the center of mass $(\mathrm{CM})$ is coincident with the geometrical center. Also, if the sphere is in contact with a nonlifted surface, the projection of the $\mathrm{CM}$ over the surface will be at the contact point. Under these conditions, the sphere will have neither acceleration nor velocity in any possible direction (i.e., the sphere is at rest) [see Fig. 1(a)].

If a sphere is built by using a nonuniform material, its $\mathrm{CM}$ would not be located at its geometrical center. In this case, when placing the sphere on a flat surface, the projection of the $\mathrm{CM}$ over that surface will not coincide with the contact point and it will overturn until reaching an equilibrium configuration [see Fig. 1(b)].

Finally, if the distribution of mass, i.e., the position of the CM, can be defined arbitrarily, the spherical system would be able to self-induce movement in any possible displacement direction (i.e., a holonomic system). That is, the basic principle of locomotion in a robotic sphere, a spherical-shaped vehicle that includes an internal mechanism which permits to vary the position of the $\mathrm{CM}$ and, therefore, to self-induce motion.

\subsection{State of the Art: Concepts and First Prototypes}

Even though robotic spheres are not widely used as mobile platforms, it is possible to find in the literature quite significant contributions to the problem, as well as new concepts and prototypes proposals. Initially, research activities were focused on validating physics concepts. In this regard, some authors have proposed different approaches where the main objective was to create a mechanical system that permits to locate the $\mathrm{CM}$ of a sphere and, therefore, to self-induce motion. Nowadays, there are basically five alternatives to reach this objective. ${ }^{12}$

The first concept is known as spring central member; ${ }^{13,14}$ this alternative has a central body that includes a driven wheel on one of its ends and a passive wheel on the other, with a spring that guarantees contact of both wheels and the spherical-shaped body [see Fig. 2(a)]. Its main disadvantage is the loss of energy due to friction between both wheels and the sphere. A similar concept, known as car driven, ${ }^{15,16,17}$ utilizes an inside vehicle to induce motion. However, this mechanism does not guarantees contact between the vehicle and the sphere [see Fig. 2(b)], which constitutes an important drawback, especially when the sphere is moving along a surface with depressions and bumps. In this case, the contact, and consequently the control over the system, may be lost.

Another alternative relies on a ballast mass, a concept that has two variants. The so-called ballast mass with fixed axis system utilizes an inner pendular mechanism that consists of two rotational degrees of freedom (DoF). ${ }^{18-27}$ The first one rotates around a fixed transverse axis and the second one around a longitudinal axis [see Fig. 2(c)]. The second variant is designated as 
(a)

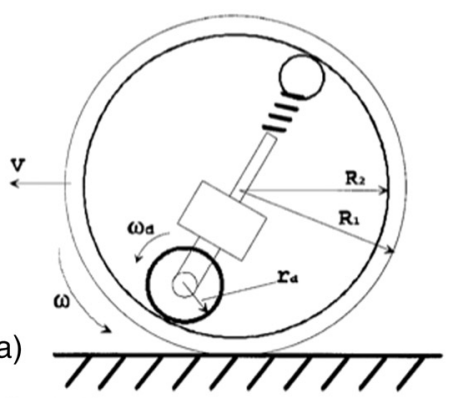

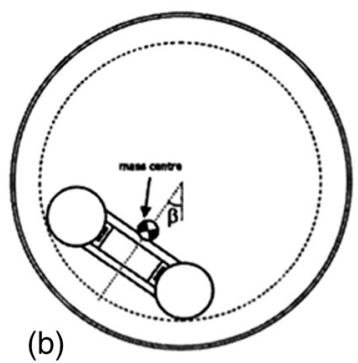

(b)

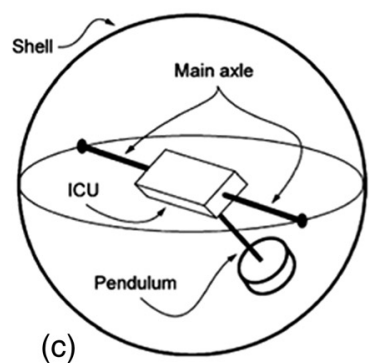

(c)

(d)
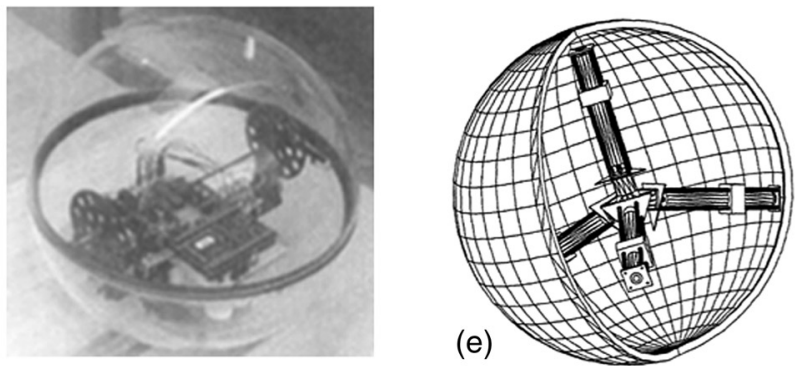

(e)

Fig. 2 Alternative mechanical systems used to self-induce motion in a robotic sphere. (a) Spring central member. (b) Car driven. (c) Ballast mass fixed axis. (d) Ballast mass moving axis. (e) Mobile masses.

ballast mass with moving axis. It also has an inner pendular mechanism, but in this case with an additional DoF that permits to move the main axis [see Fig. 2(d)].

These prototypes are examples of nonholonomic systems, since the vehicle has to start moving forward or backward in order to make turns, as it does not have the capacity to turn over itself in all possible directions. An alternative to reach the set of characteristics of holonomic vehicle is based on the mobile masses system. ${ }^{28,29}$ Prototypes using this concept take profit of the movement of masses along radial axes to modify the position of the CM [see Fig. 2(e)].

Besides these theoretical concepts, different authors have developed robotic spheres for quite different areas of applications. Perhaps, the most cited and ambitious application of robotic spheres has been the one proposed by Zhan et al. ${ }^{30}$ to explore unstructured and unknown environments by exploiting their robustness and versatility. Meanwhile, Bruhn et al. ${ }^{18}$ or Michaud et al. $^{25}$ have proposed their use for planetary exploration. Other fields of application that could benefit from these characteristics are security, surveillance, and inspection, ${ }^{10}$ whereby robotic spheres are equipped with sensors and cameras in order to facilitate the robot teleoperation.

Last but not least, since one of the principal requirements of service robots is the capacity of harmless interaction with people, robotic spheres have also been used in this area. Thus, e.g., Michaud et al. ${ }^{24,26}$ have used a robotic sphere equipped with the necessary control routines and sensors to measure child development. Children used the robot as a toy while the system acquired information to evaluate their development. Finally, more academic contributions presenting robotics spheres to study kinematics, dynamics, and control of nonholonomic systems can also be found in the literature. ${ }^{15}$

For the purposes of the present application, the selected mechanical alternative has been the ballast mass with fixed axis [Fig. 2(c)]. This mechanical option provides a fixed point (at the ends of fixed axis) where external sensors can be located. Alternatives that include internal active wheels [Figs. 2(a) and 2(b)] were discarded because of their poor energetic efficiency. The following sections discuss different aspects related to the selected mechanism, such as physics model, mechanics, and hardware and software architectures.

\subsection{Mathematical Model}

In this section, we present a synthesis of various available mathematical models that include the basic physics concepts needed to understand the system behavior. ${ }^{1,21,31}$ Since the main objective of this article is to contextualize the use of a robotic sphere in a real environment, we do not 
present a novel mathematical approach, which has been the subject of earlier contributions focusing on the development of mathematical and physics models of robotic spheres. Further information and more complex considerations can be found in Refs. 16, 19, 20, 27, 32, and 33.

The present analysis separates the system dynamics into two parts. ${ }^{32}$ A first part, inducing forward and backward motion (i.e., driving dynamics), is related to the action applied to the first DoF. The second part (i.e., steering dynamics) makes that the sphere turns and corresponds to the effect generated by the second DoF. The combined effect of these two parts endows the sphere with the characteristics of a nonholonomic vehicle.

These two parts of the mathematical model are described with more detail in the following sections.

\subsubsection{Driving dynamics}

This section summarizes the equations of motion for the driving dynamics developed through a Lagrange formulation. A deeper analysis can be found in Ref. 31, where a general case of a motion induced by a mobile mass inside a spherical body is explained. Based on this formulation, Nagai ${ }^{34}$ presented an extension for an inner pendulum-based system. Another alternative to a Lagrange formulation is a Newton formulation, first proposed for a robotic sphere by Halme et al. ${ }^{13}$

Assuming that the system is only able to move in one plane (i.e., forward and backward), the robot can be modeled as a two rigid-body system with a single DoF between them, as is shown in Fig. 3(a). The resulting Lagrange equations can be calculated as follows.

The Lagrangian is defined in Eq. (1), where $K=K_{s}+K_{p}$ and $U=U_{s}+U_{p}$ are the kinematic and potential energies of the sphere and the pendulum, respectively:

$$
\mathcal{L}=K-U \text {. }
$$

The kinematic and potential energy terms are described in Ref. 1:

$$
\begin{array}{cc}
U_{s}=0 & U_{p}=-M_{p} g l \cos \left(\theta_{s}+\theta_{p}\right) \\
K_{s}=\frac{1}{2} M_{s}\left(r \cdot \theta_{s}\right)^{2}+\frac{1}{2} J_{s} \cdot \theta_{s}^{2} & K_{p}=\frac{1}{2} M_{p}\left[\left(r \cdot \theta_{s}-l \cos \left(\theta_{s}+\theta_{p}\right)\left(\cdot \theta_{s}+\theta_{p}\right)\right)^{2}+\right. \\
& \left.+\left(l \sin \left(\theta_{s}+\theta_{p}\right)\left(\cdot \theta_{s}+\cdot \theta_{p}\right)\right)^{2}\right]+ \\
& +\frac{1}{2} J_{p}\left(\cdot \theta_{s}+\theta_{p}\right)^{2} .
\end{array}
$$

The Lagrangian motion equations can be obtained by differentiating Eq. (1) as is shown in Eq. (3), where $\tau$ is the motor torque and $\tau_{f}$ is the friction torque between the sphere and the terrain:

$$
\frac{\mathrm{d}}{\mathrm{d} t}\left(\frac{\partial \mathcal{L}}{\partial \cdot \theta_{p}}\right)-\frac{\partial \mathcal{L}}{\partial \theta_{p}}=\tau \quad \frac{\mathrm{d}}{\mathrm{d} t}\left(\frac{\partial \mathcal{L}}{\partial \cdot \theta_{s}}\right)-\frac{\partial \mathcal{L}}{\partial \theta_{s}}=-\tau+\tau_{f}
$$

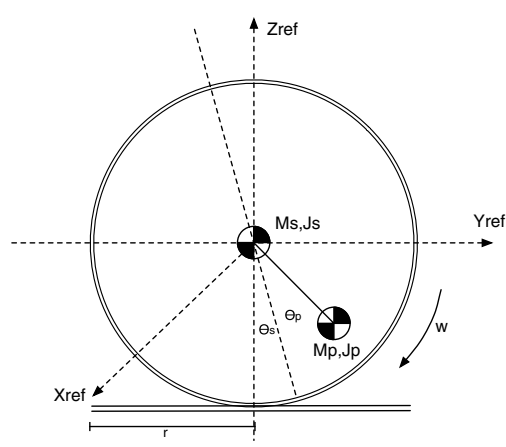

(a)

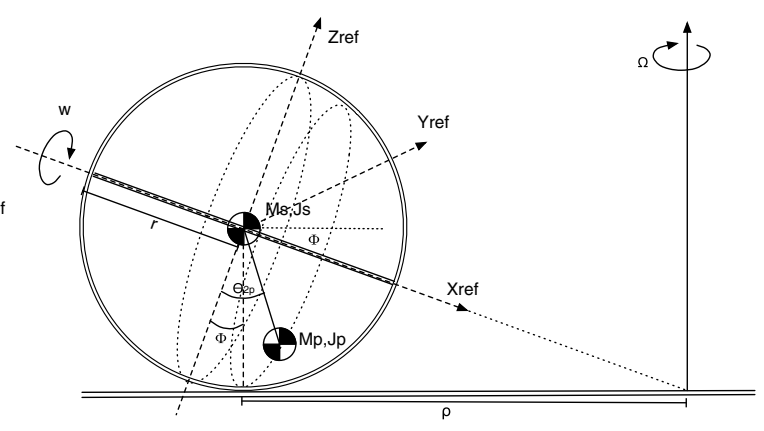

(b)

Fig. 3 Decoupled dynamics analysis. Equations are separated in motion induced by each actuator. (a) Model for forward/backward movements. (b) Model for steering movements. 
Substituting the expressions in Eq. (2) into Eq. (3) and grouping into a matrix equation, the equation of motion for a general rigid-body system can be written in the canonical form, as shown in Eq. (4). In this equation, $M(q)$ is the mass matrix, which depends on the system configuration; $\left(q=\left[\theta_{s}, \theta_{p}\right]^{T}\right)$ and $C(q, \dot{q})$ are the Coriolis terms (speed dependent); $G(q)$ is the gravity terms; $F_{\text {ext }}$ is the external forces (friction); and $\tau$ are the forces applied by the actuators:

$$
M(q) \ddot{q}+C(q, \dot{q})+G(q)-F_{\mathrm{ext}}=\tau .
$$

\subsubsection{Steering dynamics}

In this section, the second $\operatorname{DoF}\left(\theta_{2 p}\right)$; is analyzed. This angle is responsible for inducing the robot inclination, and is considered to be the robot roll angle $(\Phi)$, as shown in Fig. 3(b).

Assuming that the robot moves with a low velocity, there is an equilibrium between force and torque (including the centrifugal force of steering) and, as a result of it, the sphere follows a circumference with radius $\rho$ and an angular velocity $\Omega$. This assumption is important since a robotic sphere steering with high speed implies that Coriolis and centrifugal forces may affect predefined trajectories. A complete analysis for a high-speed conditions can be found in Refs. 31 and 34.

The radius of the turning circumference can be calculated as shown in Eq. (5), where $r$ is the radius of the sphere:

$$
\rho=\frac{r}{\tan \left(\theta_{2 p}\right)} .
$$

The angular rate for steering $(\Omega)$ can be calculated as shown in Eq. (6), where $\omega$ is the angular velocity of the robot $\left(\dot{\theta}_{\mathrm{s}}\right)$ :

$$
\Omega=\omega \cdot \sin \left(\theta_{2 p}\right)
$$

\subsection{Mechanical Design}

ROSPHERE has an inner two degrees-of-freedom pendulum. Figure 4 shows a general concept of the mechanism, including its main parts: (a) the spherical-shaped body, (b) a fixed main axis, (c) a central unit or internal control unit (ICU, as defined by other authors), and (d) the ballast or hanging mass. The first DoF allows the rotation of the ICU, and consequently of the hanging

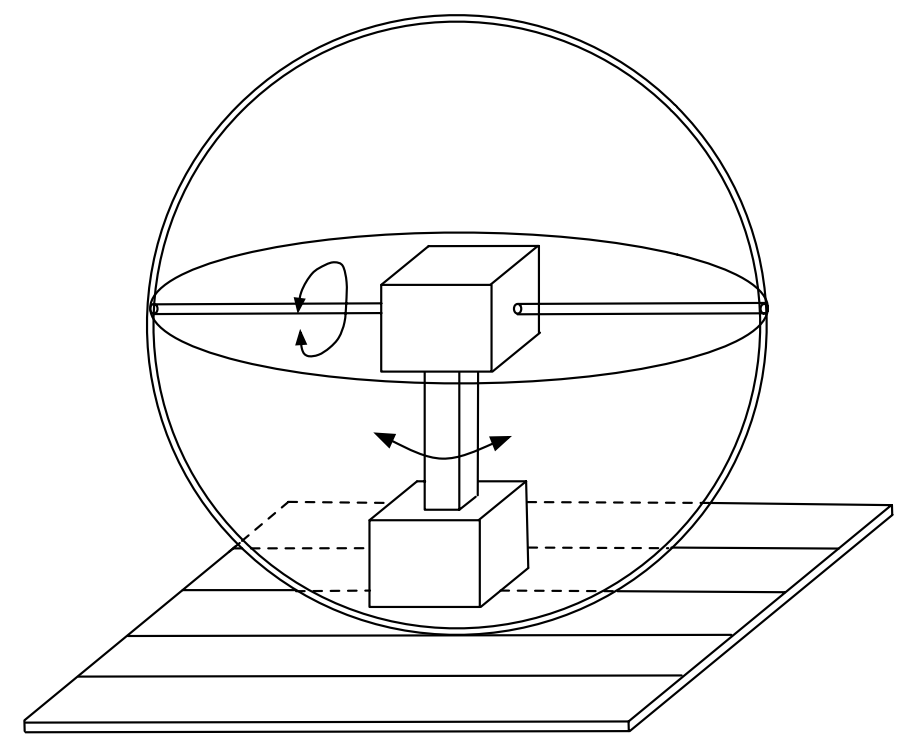

Fig. 4 ROSPHERE, internal ballast mechanism with two degrees of freedom (DoFs). The first one rotates around a fixed transverse axis, whereas the second one around a longitudinal axis. 
mass around the fixed axis. For this rotation, a continuous rotation actuator with no angle limit is needed. The second DoF, on the other hand, has a limited rotation range, which ideally should be $180 \mathrm{deg}$. However, this rotation is in practice mechanically limited. For the first prototypes, two identical servos [HS-7954SH (http://www.hitecrcd.com/products/digital/hv-ultra-premiumdigital/not-set.html)] were selected, one of which was modified to be used as a continuous rotation servo.

A first prototype, ROSPHERE v0.1, was designed to assess motion capabilities and physics concepts. A ferret ball was used as the main spherical body. This ball can be separated in two hemispheres with caps where the main aluminum axis was fixed. All the other parts of the model, including the pendulum and the ICU, were designed in a three-dimensional (3-D) modeling software (Inventor®) and built using a 3-D plastic printer.

After evaluating the results obtained with the first prototype (v0.1), some design flaws were detected that produced a certain instability of the prototype and the addition of a certain amount of useless mass to the pendulum. As explained in Sec. 3.3, another important factor is the angle required to induce motion to the system. This angle depends on different factors from which the most important one regarding the mechanics is the relative position of the $\mathrm{CM}$ with respect to the geometrical center. In other words, further the CM is from the geometrical center, the smaller the angle needed to produce motion. A second prototype, named ROSPHERE v0.2 and shown in Fig. 5, includes lighter plastic pieces enabling that the $\mathrm{CM}$ is lowered. This could be verified

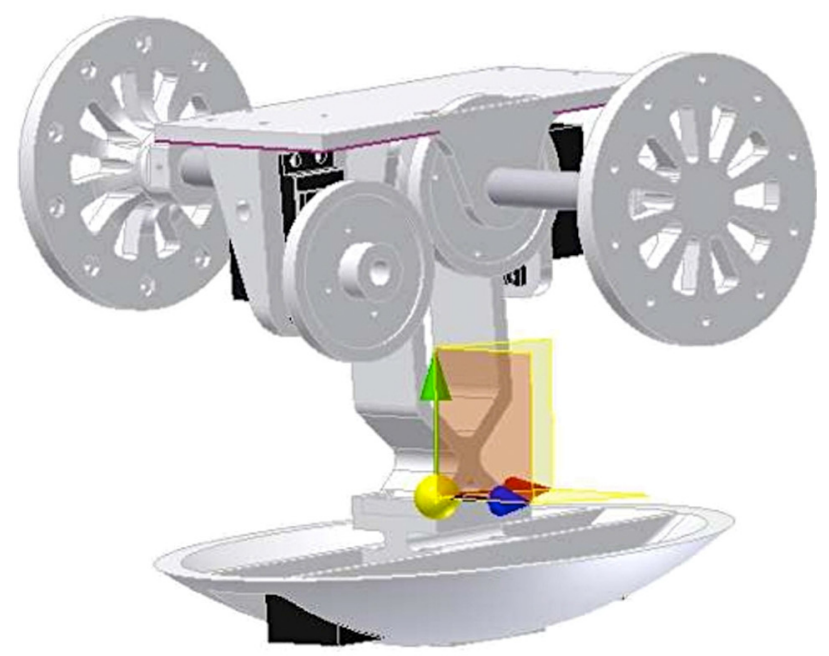

(a)

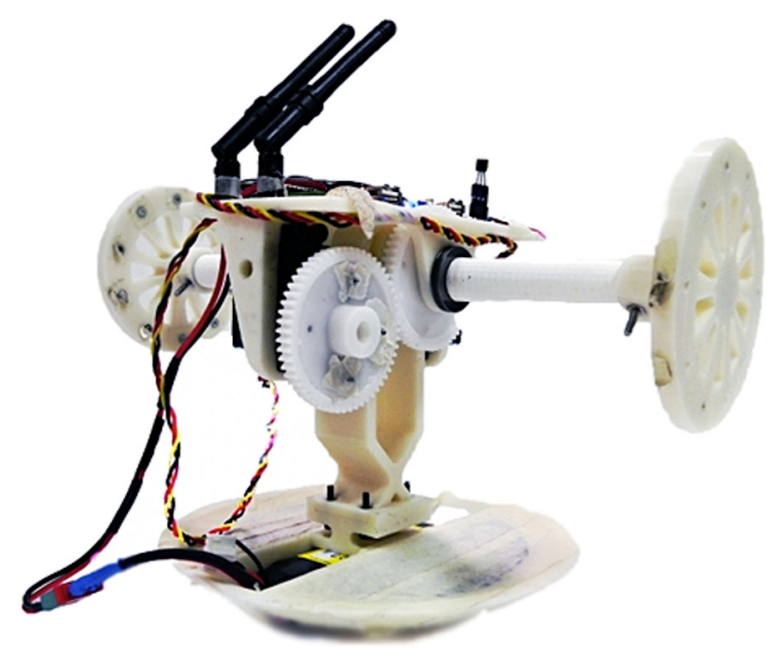

(b)

Fig. 5 Internal mechanism of ROSPHERE v0.2 uses lighter plastic pieces to lower the center of mass. (a) Mechanism designed in Inventor ${ }^{\circledR}$. (b) Real mechanism. 
before printing the new parts by comparing the position of the center of masses of both prototypes as calculated by Inventor ${ }^{\circledR}$. Besides, to stabilize the system in stationary and moving states, the servos were located in positions making the $\mathrm{CM}$ as close as possible to the pendulum body axis.

\subsection{Hardware and Software Architectures}

On the other hand, ROSPHERE is equipped with all necessary resources to behave as an autonomous vehicle. This point is nowadays at the core of the research efforts in this topic. In the earlier beginnings, the system was supplied with an embedded computing system composed by a Robovero (https://www.gumstix.com/store/product_info.php?products_id=262) (a Gumstix expansion board) and a overo fire (https://www.gumstix.com/store/product_info.php? products_id=227) (a Gumstix embedded computer) that initially used Ångström Linux. At present time, ROSPHERE v0.2 has WiFi, Bluetooth, and Xbee as communication alternatives. Furthermore, it includes other sensors, such as an inertial measurement unit (IMU), a GPS, a temperature, and relative moisture sensors. Some of these sensors can be visualized through a graphical user interface (GUI) that is part of the remote station of the robot (Fig. 6).

Robovero is an electronic board for robotic applications (http://robovero.org/) and the main board of the robot's ICU. One of its most important features is the inclusion of a microcontroller, a 9-DOF IMU (3-DOF gyroscope, 3-DOF accelerometer, 3-DOF compass), power electronics to connect motors, and a USB HUB. Robovero has a firmware that permits the microcontroller to receive commands through a USB connection. The commands allow us to read and write I/O devices (such as I2C, UARTs, SPI, PWM, A/D, etc.). Therefore, Robovero itself is not an embedded computing system, but can be considered a peripheral board. However, microntroller's commands are received through USB, either from an external computer or from an embedded one, like overo fire. Finally, the whole system (robovero + overo) is an embedded computing system which, together with the actuators and sensors, complete the hardware architecture of the system [see Fig. 7(a)].

The software system architecture can be divided into two main parts. A high-level computation layer must interpret primitive movements commands in teleoperation mode and generate the respective actuators commands or, alternatively, to navigate autonomously according to highlevel orders and the information provided by the sensors. On the other hand, there is a low-level computation layer that is in charge of collecting (reading) information from sensors and to control actuators. Both high and low layers are directly related to hardware architecture, as the high-level corresponds to overo fire programming, while the low-level corresponds to Robovero's microcontroller [see Fig. 7(b)].
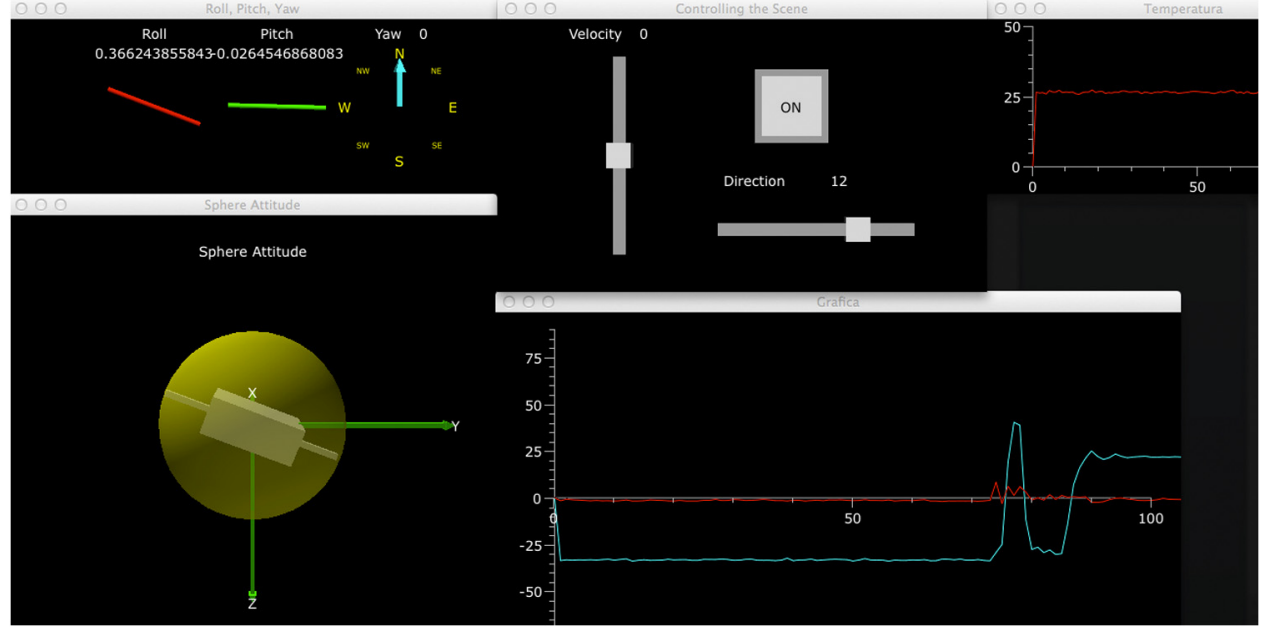

Fig. 6 Remote station graphical user interface used in teleoperation mode. It permits the control of each DoF and the visualization of the values and states of sensors, including the inertial measurement unit (roll, pitch, yaw), temperature and relative moisture. 


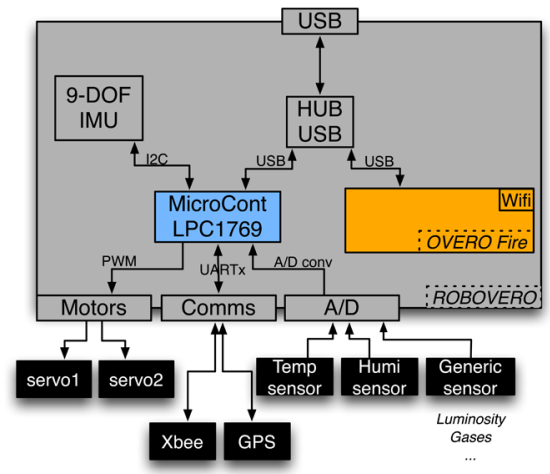

(a)
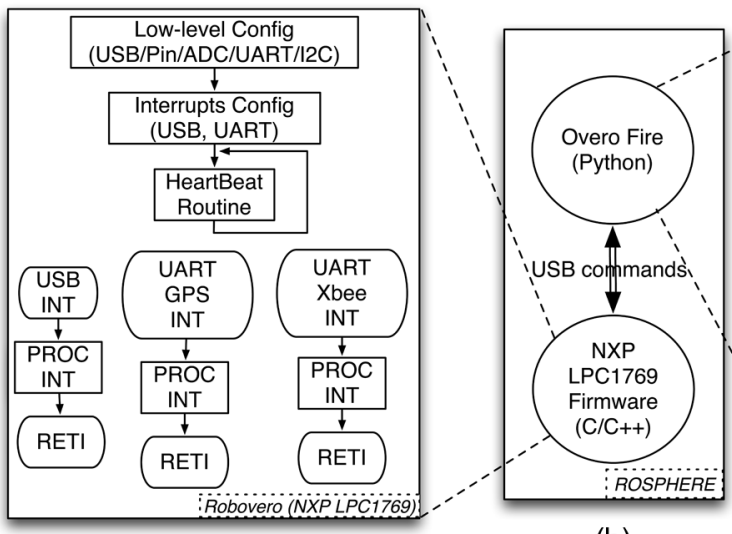

(b)

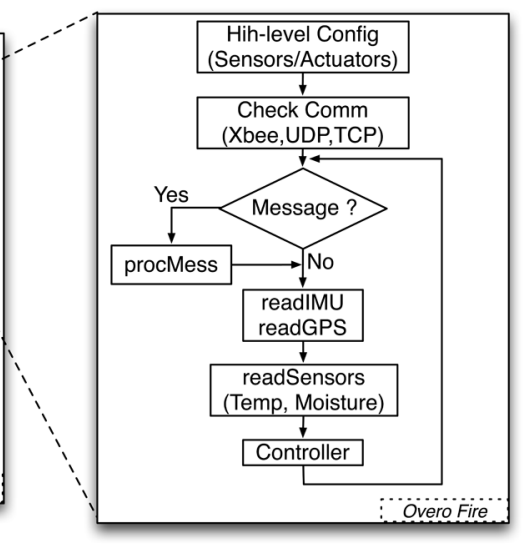

Fig. 7 ROSPHERE hardware and software architectures. (a) The hardware architecture that presents the connection between the high-level and low-level processors, sensors, and actuators. (b) The software architecture that presents the low-level and high-level computation layers.

Overo embedded computer has Linux as operating system. In a first stage of tests, Linux Ångström distribution was used to verify communication capabilities (WiFi, Bluetooth, Serial, Xbee), as well as for internal communication to Robovero's processor through USB connection. Over Linux, high-level programming is coded in Python and uses an API that wraps Robovero USB commands. Even though Python may be considered a nontime efficient programming language, it is used to control the execution flow of the main application, while time demanding parts are coded in $\mathrm{C} / \mathrm{C}++$ as extension modules. Robovero's processor, on the other hand, runs a firmware that basically is checking USB port in order to receive commands and interpret them. The original firmware was designed to read ports (I2C, UART, AD, etc.) using a polling mode. However, the firmware has been modified to accept interruptions.

\section{Experimental Results}

To validate the design and verify the capabilities of our prototype, the system needs to be thoroughly tested. A large set of experiments was designed and executed to assess the system capabilities both individually and from a global point of view. In the next subsections, four different experiments are presented, each one focused on a particular aspect of the system.

\subsection{Experiment 1: Acquisition Process}

The first scenario has been designed to validate the main concept of the system: the acquisition process and the ROSPHERE performance as a mobile sensor. Along with this line, experiment 1 has assessed the measurement ranges, including not only the maximums and minimums of the sensors but also their real acquisition rate. As well, data-location correlation and the orientation (pose) dependency have also been evaluated. 


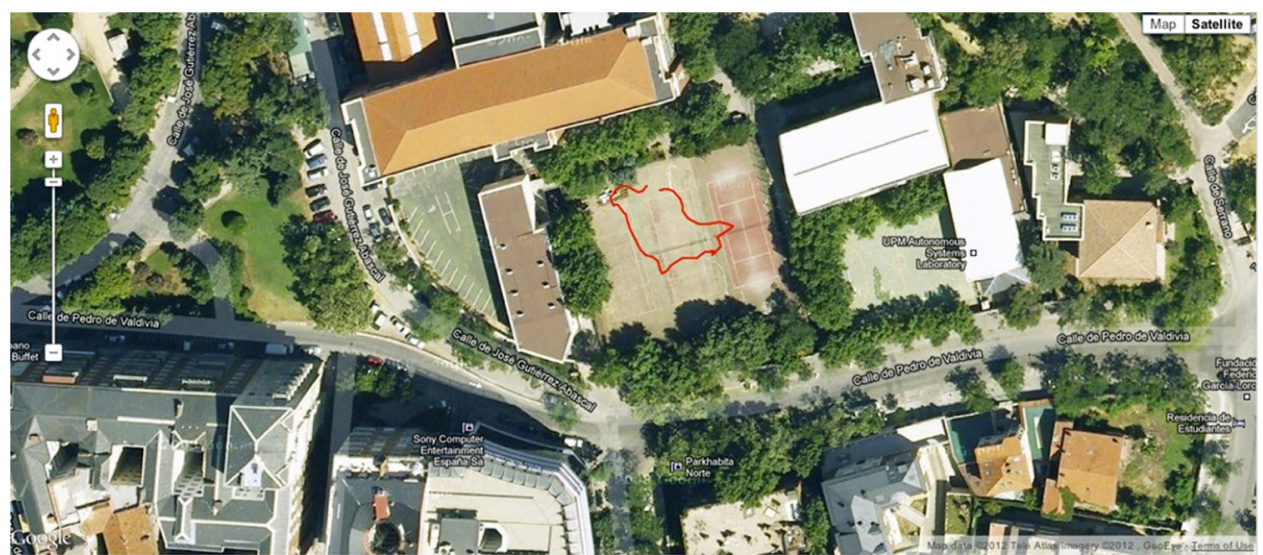

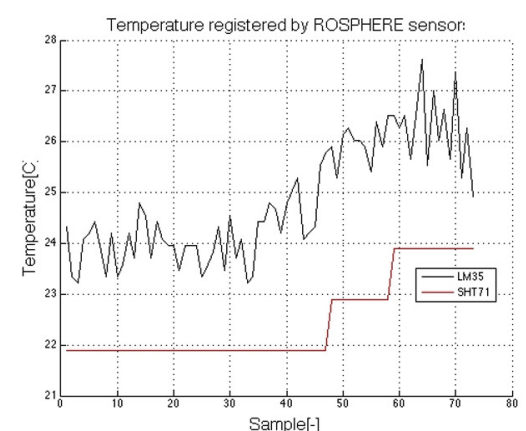

(b) (a)

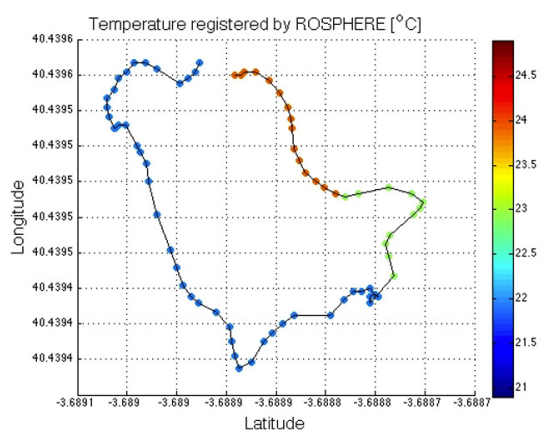

(c)

Fig. 8 Experiment 1: mobility and acquisition test in the UPM facilities. Several tests were performed in a controlled area to verify the measurement accuracy and the mobility capabilities. (a) Trajectory captured through GPS information and plotted on satellite image. (b) Comparison of the temperature acquired by both sensors $\left({ }^{\circ} \mathrm{C}\right)$. (c) Temperature along with the path covered $\left({ }^{\circ} \mathrm{C}\right)$.

It was carried out at the university facilities see Fig. 8), defining a fenced and controlled area in the sports area. Over it, several electric heaters were spread, in order to be able to set the temperature in an artificial way. Figure 8(b) illustrates this operation, where it is possible to appreciate that the temperature in this area (upper right zone) is higher than the mean one. Furthermore, in order to have a reference value, an external and a parallel measurement was performed with a precision thermometer along with the path. It is allowed to verify a maintained $11^{\circ} \mathrm{C}$ offset in the LM35 temperature sensor and $9^{\circ} \mathrm{C}$ difference in the SHT21. This error was estimated to be self-induced, provoked by the electronics and engines internal heating. Nevertheless, it has been corrected given that the tests have shown that it could be considered almost constant in steady state (around $150 \mathrm{~s}$ after the startup).

Moreover, these tests allowed to integrate both temperature sensors: while first one (SHT21) revises and attenuates the second's noise, the second one (LM35) contributes with a higher accuracy [see Fig. 8(c)]. The combination of both values provides a system with a $<1^{\circ} \mathrm{C}$ resolution. The results of this experiment have also allowed to define the ROSPHERE's maximum speed that guarantees a suitable acquisition process, as well as the minimum accuracy expected.

\subsection{Experiment 2: Safe Interaction}

The next experiment was designed to test the system's capability to safely interact with the environment and people. In order to prove that the sphere is able to work in a crowded environment with operator, machinery, and dangerous materials, the system was tested in a park. This was a good testbed of a nonstructured environment with different numerous mobile elements (i.e., persons, bicycles, cars, etc.). The main objective was to test navigation capabilities and to evaluate the impact on the environment. Other tests performed during this experiment included external 


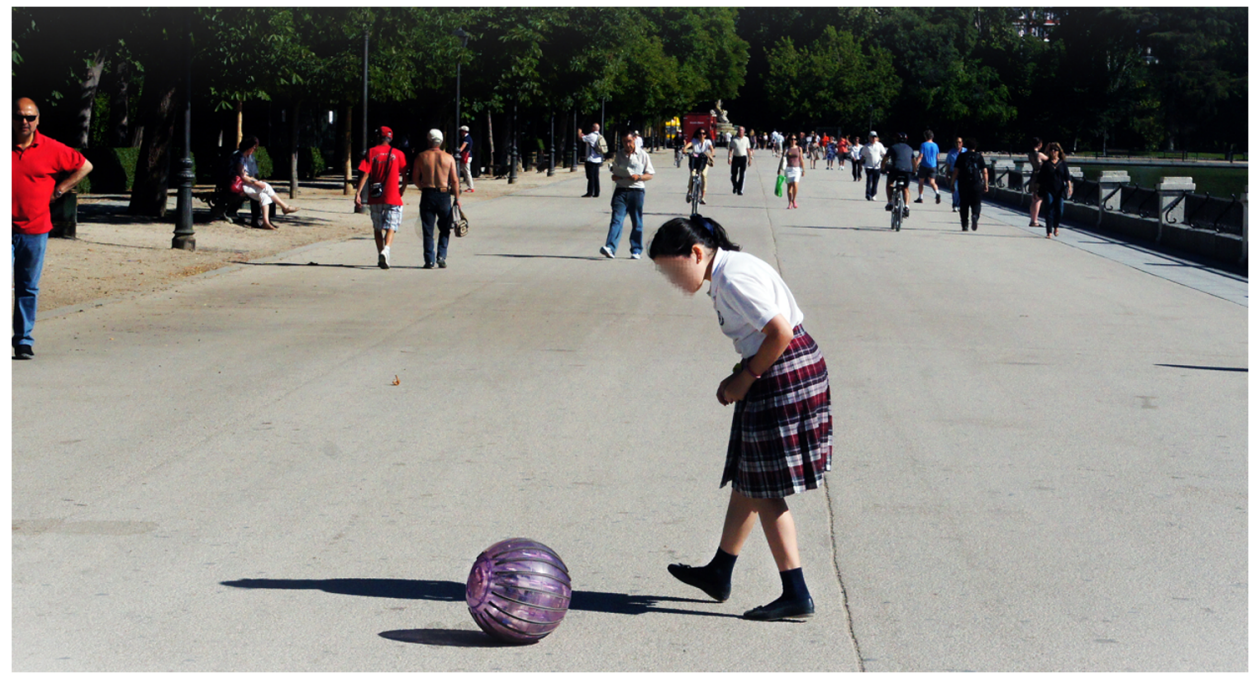

Fig. 9 ROSPHERE safely interacting with a child in El Retiro Park in Madrid, Spain.

perturbations (e.g., kids trying to play with the sphere) and minor collisions with static elements such as walls or trees. Figure 9 illustrates a safe interaction occurred during this experiment.

During this experiment other measurements were taken, although they were not the main objective of the test. In this case, correlated measurements of temperature and humidity were taken. It may be noted that the relative humidity rises near the areas where water was present, in this case around the lake and also around the fountain the robot circles. In relation

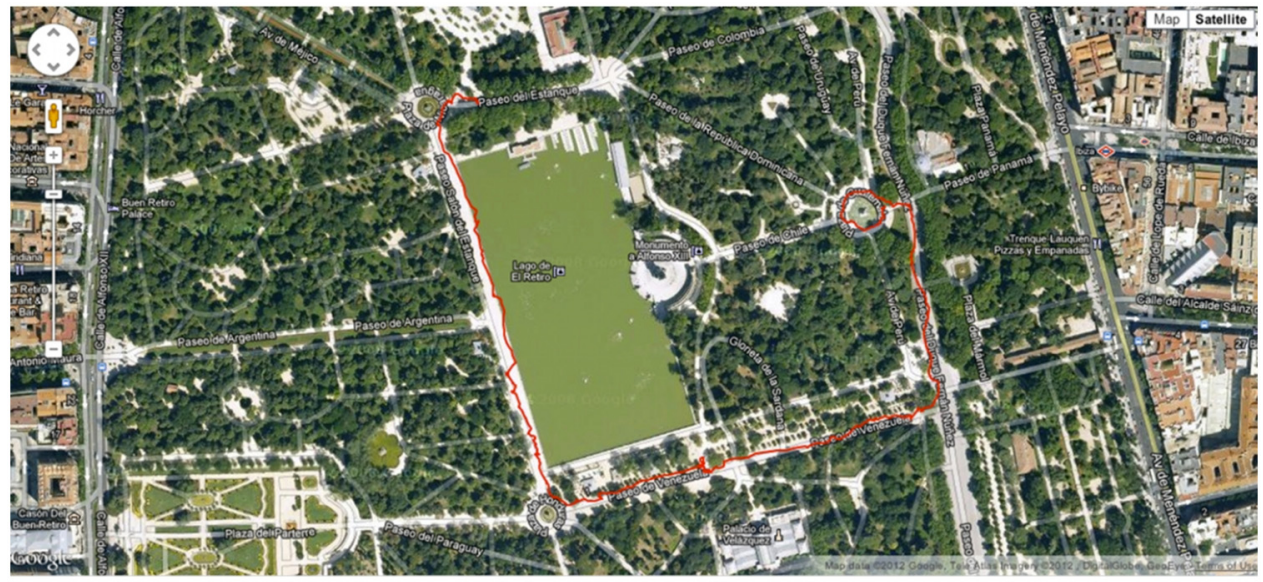

(a)

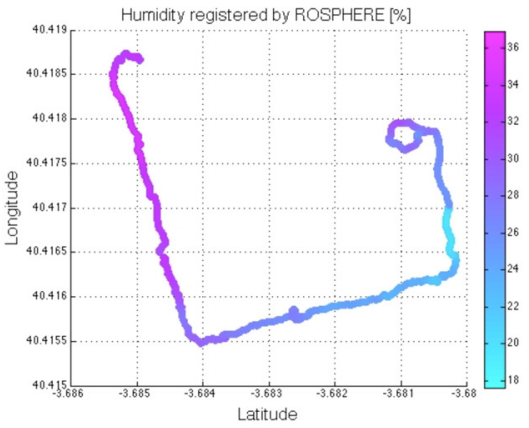

(b)

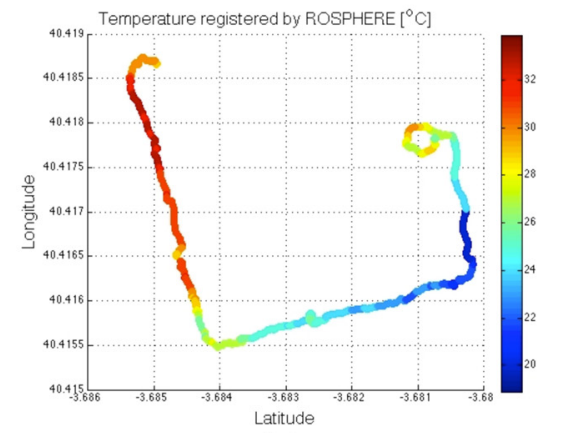

(c)

Fig. 10 Experiment 2: comparison between humidity-temperature in relation with the navigation (in El Retiro Park in Madrid, Spain). (a) Trajectory captured through GPS information and plotted on satellite image. (b) Humidity along with the path covered (\%). (c) Temperature along with the path covered $\left({ }^{\circ} \mathrm{C}\right)$. 
to the temperature, the values measured were higher around the lake because this area was more exposed to the sun that other along with the route where the robot was protected by trees and vegetation (see Fig. 10).

\subsection{Experiment 3: Terrain Conditions}

During the multiple tests carried out to assess the robot capabilities, the performance of the sphere in different terrains was also tested. Traction in different surfaces was observed. Our conclusions are that, although the velocity of the robot changed according to the terrain, ROSPHERE surpassed the expectations in any terrain. The sphere was tested in asphalt, gravel, sand, and grass as shown in Fig. 11. As mentioned before, the main differences in the performance were more noticeable in the velocity and battery consumption, but the robot never got stuck or needed help to continue moving, effectively qualifying it as all-terrain robot.

\subsection{Experiment 4: Global Performance Test in ECI Conditions}

Finally, a global performance experiment was carried out in an exterior pseudocritical infrastructure: the Automatic and Robotic Center facilities in Arganda (Madrid). It has a fenced perimeter and includes both asphalted and rugged pathways. Another feature that made this installation suitable for this test was the constant presence of operators and both autonomous and driven vehicles.

During this test, temperature and humidity were measured. Figure 12 shows the map with the robot route as well as the temperature and humidity represented by a colored line. Expected values were obtained as temperature was lower in the areas were more trees were present.

This experiment was also validated the last advantage of the spherical robot: its autonomy. The test was repeated three times, taking around 20 min each experiment. At the end, the remaining battery was higher than $40 \%$, validating the long autonomy assertion.

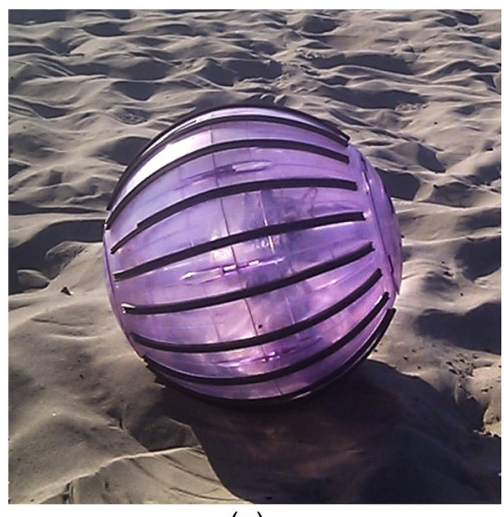

(a)

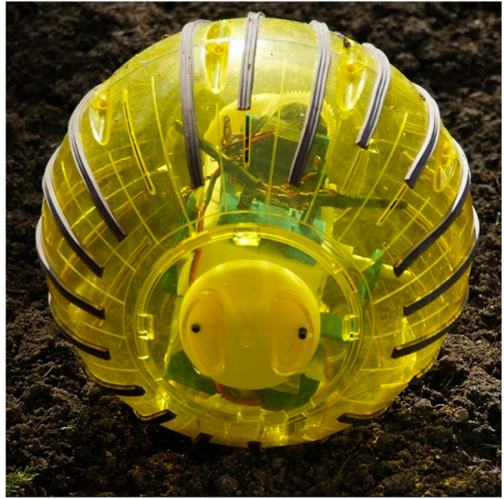

(c)

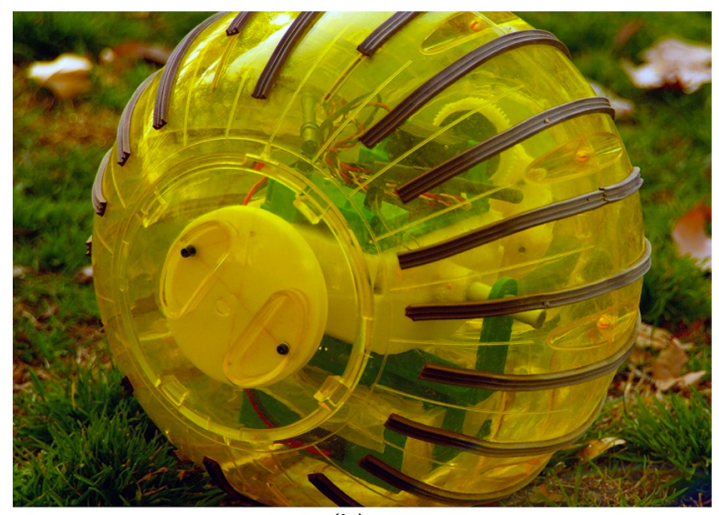

(b)

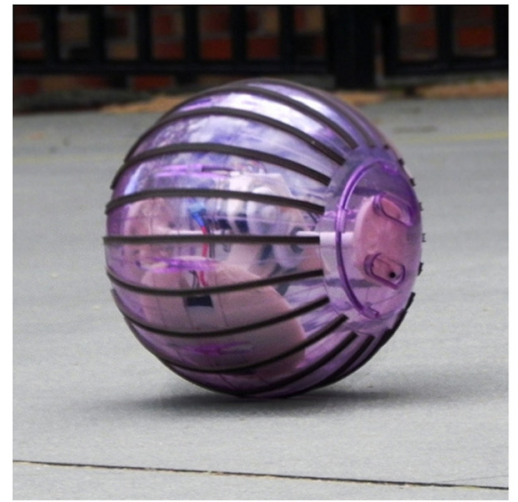

(d)

Fig. 11 Experiment 3: movement and performance test under different surfaces and terrain conditions. (a) Sandy beach. (b) Grassy park. (c) Earthy crop. (d) Pavement. 


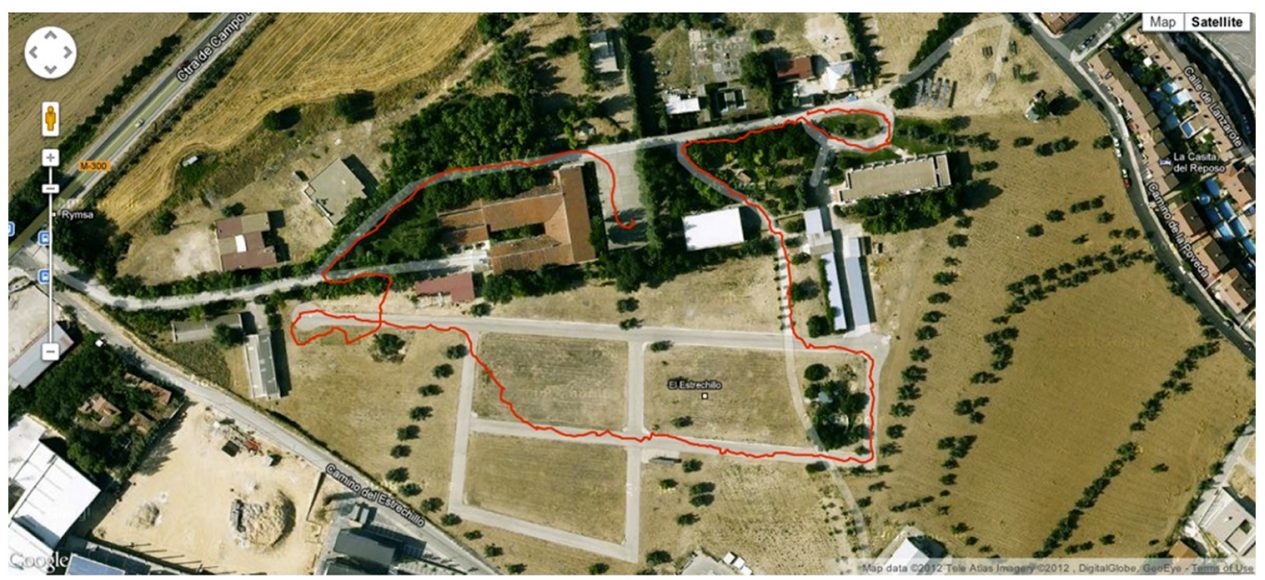

(a)

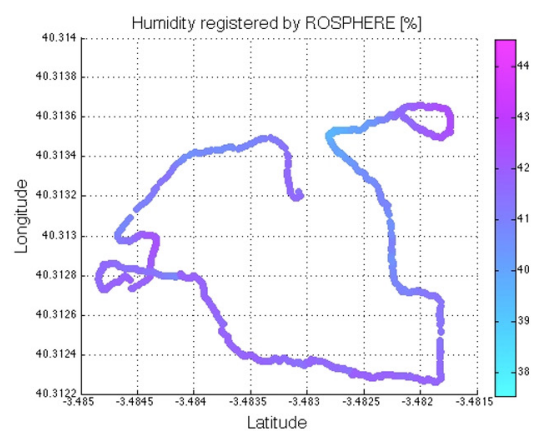

(b)

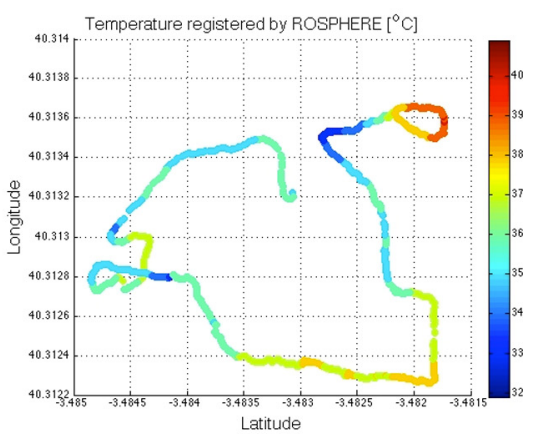

(c)

Fig. 12 Experiment 4: full simulation of an NWR facility in the CSIC installation in Arganda del Rey (Spain). Test of safety navigation, data acquisition, and surveillance task. (a) Trajectory captured through GPS information and plotted on satellite image. (b) Humidity along with the path covered (\%). (c) Temperature along with the path covered $\left({ }^{\circ} \mathrm{C}\right)$.

\section{Conclusions and Future Work}

As stated throughout this article, CIs have a crucial role for governments and companies. Due to this, in this work, the most relevant requirements for the surveillance of these scenarios have been analyzed in detail. In conclusion, a big research effort has to be made in order to improve the surveillance of these scenarios.

Currently, the most common solutions are combinations of static sensors (i.e., cameras and motion detection) and human guards. Unfortunately, these systems are generally tailored and not flexible enough. Modern MRSs (aerial and ground) devoted to perform surveillance would improve this situation.

This article presents the main aspects related to the design, construction, and implementation of ROSPHERE, a spherical-shaped robot that combines the reliability of the wheeled robot with the flexibility and versatility required to operate in different types of terrains. Due to this, it turns out to be an excellent candidate to be a part of a heterogeneous robotic team for surveillance.

These features are mainly achieved due to its original movement based on center of gravity destabilization instead of using friction-based movement. Additionally, its shape and weight prevent ROSPHERE from damaging the environment or people, being able to continue with its moving and sensing capabilities after collisions or even small falls.

However, some issues have to be addressed in order to fulfill fully autonomous operation and integration into a heterogeneous system. Thus, in order to improve the control performance, the addition of a rotational speed sensor would allow to control the sphere in extreme slippery surfaces. Furthermore, a wireless link for external sensors will enhance the data accuracy and make the connectivity easier.

Finally, in order to make easier the integration of ROSPHERE into a complex system, an effort is being carried out so as to provide it with a standard connectivity by using a common 
framework such as robot operating system. This task becomes easier since it natively works using Ubuntu as its operating system.

Briefly, ROSPHERE has been validated as a suitable alternative for accurate measurements in CI. It has been proved that it can be a good replacement for some tasks in ECI surveillance, as well as for outdoor scenarios in general.

\section{Acknowledgments}

This work has been supported by the Robotics and Cybernetics Research Group at Universidad Politécnica de Madrid (Spain), and funded under the projects "ROTOS: MultiRobot system for outdoor infrastructures protection," sponsored by Spain Ministry of Education and Science (DPI2010-17998), and the project ROBOCITY 2030 project, sponsored by the Community of Madrid (S-0505/DPI/000235).

\section{References}

1. European Defense Agency, Communication from the Commission on a European Programme for Critical Infrastructure Protection, http://eurlex.europa.eu/LexUriServ/site/ en/com/2006/com2006_0786en01.pdf (2006).

2. T. Moore, "Robots for nuclear power plants," IAEA Bullet. (1985).

3. F. E. Gelhaus and H. T. Roman, "Robot applications in nuclear power plants," Prog. Nucl. Energy 23(1), 1-33 (1990), http://dx.doi.org/10.1016/0149-1970(90)90012-T.

4. A. Birk and H. Kenn, "Roboguard, a teleoperated mobile security robot," Control Eng. Pract. 10(11), 1259-1264 (2002), http://dx.doi.org/10.1016/S0967-0661(02)00088-6.

5. P. E. Rybski et al., "A team of robotic agents for surveillance," in Proc. 4th Int. Conf. Autonomous Agents (AGENTS '00), pp. 9-16, ACM, New York, NY, USA (2000).

6. A. Talukder et al., "Real-time detection of moving objects in a dynamic scene from moving robotic vehicles," in Proc. IEEE/RSJ Int. Conf. Intelligent Robots and Systems (IROS 2003), Las Vegas, Nevada, Vol. 2, pp. 1308-1313, IEEE (2003).

7. C.-H. Huang et al., "A hybrid moving object detection method for aerial images," Lec. Notes. Comput. Sci. 6297, 357-368 (2010), http://dx.doi.org/10.1007/978-3-642-15702-8.

8. G. R. Rodríguez-Canosa et al., "A real-time method to detect and track moving objects (DATMO) from unmanned aerial vehicles (UAVs) using a single camera," Remote Sens. 4(12), 1090-1111 (2012), http://dx.doi.org/10.3390/rs4041090.

9. A. Miller et al., "Person tracking in uav video," Lec. Notes Comput. Sci. 4625, 215-220 (2008), http://dx.doi.org/10.1007/978-3-540-68585-2.

10. M. Seeman et al., "An autonomous spherical robot for security tasks," in Proc. IEEE International Conference on Computational Intelligence for Homeland Security and Personal Safety, Alexandria, Virginia, pp. 51-55, IEEE (2006).

11. Q. Zhan et al., "Analysis of moving capability of a spherical mobile robot," J. Beijing Univ. Aeronaut. Astron. 31(7), 744-747 (2005).

12. R. H. Armour and J. F. V. Vincent, "Rolling in nature and robotics: a review," J. Bionic Eng. 3(4), 195-208 (2006), http://dx.doi.org/10.1016/S1672-6529(07)60003-1.

13. A. Halme, T. Schönberg, and Y. Wang, "Motion control of a spherical mobile robot," in Proc. 4th Int. Workshop on Advanced Motion Control (AMC'96 MIE), Mie University, Tsu-City, Japan, pp. 259-264, IEEE (1996).

14. A. Halme et al., "A spherical mobile micro-robot for scientific applications," in Proc. 4th ESA Workshop on ASTRA, pp. 321-327 (1996).

15. A. Bicchi et al., "Introducing the "SPHERICLE": an experimental testbed for research and teaching in nonholonomy," presented at the IEEE International Conference on Robotics and Automation, Vol. 3, pp. 2620-2625 (1997), http://dx.doi.org/10.1109/ROBOT.1997 .619356 .

16. C. Camicia, F. Conticelli, and A. Bicchi, "Nonholonomic kinematics and dynamics of the sphericle," in Proc. IEEE/RSJ Int. Conf. Intelligent Robots and Systems (IROS 2000), Takamatsu, Japan, Vol. 1, pp. 805-810, IEEE (2000). 
17. J. Alves and J. Dias, "Design and control of a spherical mobile robot," Proc. Inst. Mech. Eng., Part C 217(6), 457-467 (2003), http://dx.doi.org/10.1.1.119.1935.

18. F. C. Bruhn et al., "A preliminary design for a spherical inflatable microrover for planetary exploration," Acta Astronaut. 63(5), 618-631 (2008), http://dx.doi.org/10.1016/j.actaastro .2008.01.044.

19. Y. Cai, Q. Zhan, and C. Yan, "Two-state trajectory tracking control of a spherical robot using neurodynamics," Robotica, 30(2), 195-203 (2011), http://dx.doi.org/10.1017/ S0263574711000518.

20. Q. Jia, H. Sun, and D. Liu, "Analysis of actuation for a spherical robot," in IEEE Conf. on Robotics, Automation and Mechatronics, Pasadena, California, pp. 266-271, IEEE (2008).

21. J.-F. Laplante, "Etude de la Dynamique D'un Robot Spherique et de son effet sur L'attention et la Mobilite de Jeunes Enfants," Master's Thesis, Université de Sherbrooke, Sherbrooke (Québec), Canada (2004).

22. J.-F. Laplante, P. Masson, and F. Michaud, "Analytical longitudinal and lateral models of a spherical rolling robot," 1-6 (2007).

23. D. Liu, H. Sun, and Q. Jia, "Stabilization and path following of a spherical robot," in IEEE Conf. Robotics, Automation and Mechatronics, Chengdu, China, pp. 676-682, IEEE (2008).

24. F. Michaud and S. Caron, "Roball, the rolling robot," Auton. Robots 12(2), 211-222 (2002), http://dx.doi.org/10.1023/A:1014005728519.

25. F. Michaud, J. de Lafontaine, and S. Caron, "A spherical robot for planetary surface exploration," in Proc. 6th Int. Symposium on Artificial Intelligence and Robotics \& Automation in Space, Quebec, Canada (2001).

26. F. Michaud et al., "Autonomous spherical mobile robot for child-development studies," IEEE Trans. Syst., Man Cybern., Part A 35(4), 471-480 (2005), http://dx.doi.org/10 .1109/TSMCA.2005.850596.

27. Q. Zhan et al., "Dynamic trajectory planning of a spherical mobile robot," in IEEE Conf. Robotics, Automation and Mechatronics, Bangkok, Thailand, pp. 1-6, IEEE (2006).

28. A. Amir Homayoun Javadi and P. Mojabi, "Introducing August: a novel strategy for an omnidirectional spherical rolling robot," in Proc. IEEE Int. Conf. Robotics and Automation, Washington, DC, Vol. 4, pp. 3527-3533, IEEE (2002).

29. S. Shengju et al., "Modeling and simulation of a spherical mobile robot," Comput. Sci. Inf. Syst. 7(1), 51-62 (2010), http://dx.doi.org/10.2298/CSIS1001051S.

30. Q. Zhan, Y. Cai, and Z. Liu, "Near-optimal trajectory planning of a spherical mobile robot for environment exploration," in IEEE Conf. Robotics, Automation and Mechatronics, Chengdu, China, pp. 84-89, IEEE (2008).

31. A. Koshiyama and K. Yamafuji, "Design and control of an all-direction steering type mobile robot," Int. J. Robot. Res. 12(5), 411-419 (1993), http://dx.doi.org/10.1177/ 027836499301200502

32. E. Kayacan, Z. Bayraktaroglu, and W. Saeys, "Modeling and control of a spherical rolling robot: a decoupled dynamics approach," Robotica 30(4), 671-680 (2012), http://dx.doi.org/ $10.1017 / \mathrm{S} 0263574711000956$.

33. D. Liu et al., "Motion control of a spherical mobile robot by feedback linearization," in 7th World Congress on Intelligent Control and Automation (WCICA 2008), Chongqing, China, pp. 965-970, IEEE (2008).

34. M. Nagai, "Control system for a spherical robot," Master's Thesis, Luleå Tekniska Universitet (2008).

Juan David Hernández obtained the electronics engineering (BSc equivalent) degree from Pontificia Universidad Javeriana in Cali, Colombia and his MSc in automation and robotics from Universidad Politécnica de Madrid. Currently, he is a $\mathrm{PhD}$ student at the Underwater Robotics Research Center at the University of Girona. His research interests include mechanics theories (kinematics and dynamics), algorithms for modeling and simulation of serial/branched rigid-body robotic systems, and motion planning algorithms for underwater robots.

David Sanz has a BSc degree in telecommunication engineering and an MSc degree in robotics, both from Universidad Politécnica de Madrid. Currently he is working on his PhD thesis, as a 
researcher at the Robotics and Cybernetics Research Group of the Centre for Automation and Robotics, Technical University of Madrid-Spanish National Research Council. His research interests include autonomous aerial vehicles, obstacle \& avoidance systems and cognitive architectures.

Gonzalo R. Rodríguez-Canosa received his MSc degree in automatics and robotics from the Technical University of Madrid and industrial engineer from the University of Seville. He is interested in MultiRobot Systems (MRS) and decentralized cooperation for the development of surveillance MRS for Critical Infrastructures.

Jorge Barrientos is studying for an electronics and automation engineering degree at Universidad Politécnica de Madrid. Since 2011, he has been a member of the Robotics and Cybernetics research group of the Centre for Automation and Robotics, Technical University of Madrid-Spanish National Research Council.

Jaime del Cerro received the Msc Engineer degree in automatic and electronic in 1996 and the $\mathrm{PhD}$ in robotics in 2007 from Universidad Politécnica de Madrid. He is professor of control theory at Universidad Europea de Madrid. His research is focused on GNC systems and multirobot systems applied to both aerial and ground vehicles.

Antonio Barrientos received the Msc Engineer degree in automatics and electronics in 1982 and a $\mathrm{PhD}$ in robotics in 1986, both from Universidad Politécnica de Madrid. In 2002 he obtained an MSc degree in biomedical engineering from Universidad Nacional de Educación a Distancia. He has been professor of Robotics, Computers and Control Engineering at Universidad Politécnica de Madrid since 1988 and has been working for more than 30 years in robotics, developing industrial and service robots. Currently his main interest areas are aerial and field robotics. $\mathrm{He}$ is author of several textbooks in robotics and manufacturing automation, and coauthor of white books on the state of robotics and manufacturing in Spain. He is also coauthor of more than 100 scientific papers in journals and conferences. He has been reviewer of several international journals and currently is editorial board member of the International Journal of Advanced Robotic Systems. Currently, he is the head of the Robotics and Cybernetics research group of the Centre for Automation and Robotics, Technical University of Madrid-Spanish National Research Council, and a senior member of the IEEE. 